us, compared with $60 \%$ in the intervention group $[\mathrm{OR}=2.05$ (95\% CI: 1.60, 2.62)]. In the intervention group, more children were transferred to CCR $52 \%$ vs $32 \%$ in the control $[\mathrm{OR}=1.7$ (CI: 1.3-2.41)], $65 \%$ of the mothers in intervention group reached at CCR vs $57 \%$ of the mothers in the control group $[\mathrm{OR}=1.69 \mathrm{CI}: 1.27-2.41)]$ and returned to receive the PCR result of their child, $6.7 \%$ in the control vs $8.2 \%$ in intervention $[\mathrm{OR}=2.3$ (CI: $1.36,3.87)]$.

Conclusion The intervention had a greater impact on the number of visits to CPP, the transfer of mothers from CPP to CCR, and the reception of PCR results in CCR by the companion.

\section{PO 8300 REGIONAL CENTER FOR REGULATORY EXCELLENCE IN CLINICAL TRIAL OVERSIGHT - TRAINING 2017}

Delese Darko*, Yvonne Adu-Boahen. Food and Drugs Authority, Accra, Ghana

\subsection{6/bmjgh-2019-EDC.71}

Background The competencies of the various national medicines regulatory agencies (NMRAs) in Africa vary which leads to generally porous regulatory systems for clinical trial oversight. Consequently, many trials have been conducted under unacceptable conditions compromising participants' safety and data credibility and resulted in questionable outcomes that are used for making scientific judgement in addressing issues of public health in Africa.

To improve the safety and quality of health technologies in Africa, the New Partnership for African Development (NEPAD) agency launched a programme to designate Regional Centres of Regulatory Excellence (RCOREs) with the specific objective of bridging existing gaps between African NMRAs through strengthening regulatory capacity of African Union member states. The Food and Drugs Authority (FDA), Ghana, was designated as RCORE for Clinical Trials oversight in May 2014.

Methods To achieve the RCORE objectives, the FDA collaborated with the School of Public Health (SPH), University of Ghana to develop a training manual and piloted a training programme with funds from the International AIDS Vaccine Initiative (IAVI) through NEPAD.

The programme, consisting of 4 compulsory modules, was organised from 6-30 November 2017 for 10 participants from Zambia, Sierra Leone, Liberia, Rwanda and Ghana. Interactive training methods in the form of theoretical and practical sessions were employed.

Results The pilot RCORE training was successful with expected training objectives achieved. Participants gained hands-on experience through activities like observing Good Clinical Practice inspection and a Technical Advisory Committee Meeting. Participants were given template tools to assist in developing regulatory guidelines and forms in their respective countries.

A follow-up questionnaire was circulated to participants to assess the impact of the training on their work. Feedback indicates that regulation of clinical trials has improved in their respective institutions.

Conclusion This pilot fellowship training was successful, leading to the improvement of clinical trial regulation in the participating countries.

\section{PO 8302 IMPACT OF TWO ANNUAL ROUNDS OF MASS DRUG ADMINISTRATION WITH DIHYDROARTEMISININ- PIPERAQUINE ON MALARIA TRANSMISSION IN A PROSPECTIVE COHORT STUDY}

2Julia Mwesigwa*, ${ }^{2}$ Jane Achan, ${ }^{2}$ Miriam Wathuo, ${ }^{2}$ Archibald Worwui, ${ }^{2}$ Nuredin Mohammed, ${ }^{2}$ Fatomatta Kanuteh, ' Jean-Pierre Van Geertruyden, ${ }^{2}$ Umberto D Alessandro. ${ }^{1}$ Faculty of Medicine and Health Sciences, University of Antwerp, Belgium; ${ }^{2}$ MRC Unit The Gambia at the London School of Hygiene and Tropical Medicine, Banjul, The Gambia

\subsection{6/bmjgh-2019-EDC.72}

Background Mass drug administration (MDA) may reduce malaria transmission in low-transmission areas and interrupt transmission. The impact of MDA with dihydroartemisininpiperaquine (DP) on malaria infection and clinical malaria was determined in a prospective cohort study in The Gambia.

Methods Single annual MDA rounds with DP were done in 2014 and 2015 in a prospective cohort among residents aged $>6$ months in twelve villages in The Gambia at the start of the transmission season in June. Monthly blood samples for microscopy and PCR were collected during the transmission season from July to December, post MDA and once before MDA during the dry season in April. The incidence of infection and clinical malaria post-MDA were compared to 2013 and mixed effects logistic regression models assessed the efficacy and risk of re-infection post MDA.

Results Coverage of 3 DP doses was $68.22 \%$ in 2014 and $65.60 \%$ in 2015 . Compliance to 3 doses was high, $83.11 \%$ in 2014 and $85.93 \%$ in 2015. Incidence of infection in 2014 (2014: IR=0.23 PPY, 2013: IR=1.12 PPY, $p<0.01$ ) and clinical malaria in 2014 (2014: IR=0.08 PPY, 2013: IR=0.39: $\mathrm{IRR}=0.22, \quad \mathrm{p}<0.01) \quad$ and $2015 \quad(2015: \quad \mathrm{IR}=0.19, \quad 2013$ : $\mathrm{IR}=0.38, \quad \mathrm{IRR}=0.50, \mathrm{p}<0.01$ ) was significantly lower after MDA compared to 2013. The incidence of clinical malaria remained higher in eastern Gambia compared to the western region. Subjects that took 3 DP doses had lower odds of infection in 2014 at 28 days $(\mathrm{OR}=0.61,95 \% \mathrm{CI}$ : $0.38-0.99)$ and 42 days (2014: $\mathrm{OR}=0.52$, 95\% CI: $0.29-0.89$ )

Conclusion A single annual MDA round with DP temporarily reduced malaria infection and clinical disease during the transmission season and subjects that took 3 doses had lower risk of infection. However, several MDA rounds covering the entire transmission season and some targeting the human reservoir during the dry season, are needed to achieve a more marked sustained reduction of transmission.

\section{PO 8313 PREDICTORS OF HEALTH INSTITUTIONAL READINESS FOR EBOLA EPIDEMIC CONTAINMENT IN NIGERIA: A STRUCTURAL EQUATION MODELLING (SEM) APPROACH}

${ }^{1}$ Joshua Adekeye*, ${ }^{1}$ Eustasius Musenge, ${ }^{2}$ Oyedunni Arulogun. ' University of the Witwatersrand, Johannesburg, South Africa; ${ }^{2}$ University of Ibadan, Ibadan, Nigeria

\subsection{6/bmjgh-2019-EDC.73}

Background There is paucity of literature on organisational readiness in the health space. Previous studies focus on epidemic preparedness and often depict readiness as a minor element in the implementation space. This study investigated the predictors of health institutional readiness to implement innovations for combating an Ebola epidemic outbreak in Nigeria. 
Methods The cross-sectional, mixed methods design was employed. The study population was 785 health care workers and non-health workers working at University of Port-Harcourt Teaching Hospital (Nigeria). The purposive sampling was used for qualitative study while the stratified random sampling technique was utilised for the quantitative study. Qualitative data were collected from fifteen respondents while a total of 511 questionnaires were administered at the study site. The qualitative data was analysed using inductive thematic analysis. The quantitative data was analysed using structural equation modelling (SEM).

Results The qualitative study suggested that quality improvement was perceived as most useful in influencing all the tree sub-components of readiness. Training is perceived as most useful in building readiness while it is perceived to be moderately useful in influencing the sub-component of readiness. The OLS estimates indicates that QI/QA exert a positive and significant effect on motivation $(\beta=0.004, p<0.05)$ and general capacity score $(\beta=0.28 \mathrm{p}<0.05)$ while it inversely but significantly exerts influence on innovation specific capacity $(\beta=-0.21 \times 10-3, p<0.05)$. The $\mathrm{SEM} /$ pathway analysis shows the direct and indirect routes of interactions among predictors of readiness after adjusting for confounders. All the explanatory variables have significant effect on readiness except gender which was dropped from the final model.

Conclusion The strength of evidence of how an evidencebased system for innovation support can influence readiness was established. Though readiness is a rate-determining step in ensuring robust and effective implementation outcomes for epidemic containment, exploring innovation outcomes and their amplification through explicitly target readiness dynamics requires further investigation.

\section{PO 8355 HOST GENETIC POLYMORPHISMS AND ASYMPTOMATIC MALARIA IN SOUTHERN GHANA}

${ }^{1}$ Paulina Otu*, ${ }^{2}$ Aminata Colle Lo, ${ }^{2}$ Ben Gyan, ${ }^{1}$ Yaw Afrane, ${ }^{2}$ Linda Eva Amoah. ${ }^{1}$ University of Ghana, Legon, Accra, Ghana; ${ }^{2}$ Immunology Department, Noguchi Memorial Institute for Medical Research, College of Health Sciences, University of Ghana, Accra, Ghana

\subsection{6/bmjgh-2019-EDC.74}

Background Despite several interventions through malaria control programmes, asymptomatic malaria is a major barrier to control as asymptomatic individuals serve as reservoirs from which others are re-infected. The mechanism by which these individuals remain asymptomatic is not well understood. Much work has been done in relation to human genes and their association to severe, mild and uncomplicated malaria. However, there is limited knowledge regarding host genetic factors and asymptomatic malaria.

Method In this study, we investigated the association between host genetic polymorphisms of glucose-6-phosphate dehydrogenase gene (G6PD), mannose binding lectin (MBLG54A), tumor necrotic factor alpha (TNF-G308A) and nitric oxide synthase 2 (NOS2-G954C) and the outcome of asymptomatic P. falciparum malaria in 150 healthy individuals in southern Ghana.

Results We found a significant association between G6PDd and asymptomatic malaria with a prevalence of $9.6 \%$ $(\mathrm{p}=0.035$, by chi-square test). All the individuals who were heterozygous and hemizygote deficient (5.3\% and $4.3 \%)$ were found to be asymptomatic. Individuals homozygous (GG) for TNF (G308A) were found to be highly asymptomatic $(\mathrm{p}=0.019$, by chi-square test). Regarding MBL (G54A) and
NOS (G954C), no significant association was found between these markers and asymptomatic malaria.

Conclusion Upon reviewing our data with other data from published work, we conclude that both heterozygous and hemizygous individuals with G6PD A- and homozygous individuals (GG) of TNF (G308A) polymorphisms could be predisposed genetically to asymptomatic malaria.

\section{PO 8356 ANGIOGENIC AND ANGIOSTATIC FACTORS IN THE SALIVA OF MALARIA PATIENTS}

${ }^{1}$ Cecilia Lekpor*, ${ }^{1}$ Felix Botchway, ${ }^{2} J o n a t h a n$ Stiles, ${ }^{2}$ Wilson 0 Nana. ${ }^{1}$ Department of Patholgy, University of Ghana, Accra, Ghana; ${ }^{2}$ Morehouse School of Medicine, Atlanta (GA), USA

\subsection{6/bmjgh-2019-EDC.75}

Background Malaria mortality is associated with exaggerated host responses to inflammatory factors such as C-X-C motif chemokine 10 (CXCL10) and host biomarkers such as angiopoietin 1 (Ang-1) and angiopoietin 2 (Ang-2). The aim of this study was to determine saliva levels of CXCL10, Ang-1 and Ang-2 and compare with plasma levels regarding their potential as biomarkers of malaria, which may be useful for further development of highly efficient non-invasive malaria detection methods.

Methods Case control study involving 213 subjects (119 with and 94 without malaria) aged 1-16 years. Haematological determination was done using Haematology Analyser. Plasmodium Lactate Dehydrogenase/Histidine Rich Protein-2 (pLDH/ HRP-2) Antigen rapid diagnostic test (RDT) were performed. Plasma and saliva levels of CXCL10, Ang-1 and Ang-2 were measured using Elisa kit. Data was presented as mean \pm standard error or median and interquartile range (IQR). A p-value $<0.001$ was considered statistically significant. Results There was decreased plasma levels of Ang-1 and increased plasma levels of CXCL10 and Ang-2 in individuals with malaria compared to those without malaria (Ang-1, $\mathrm{p}<0.009$; Ang-2, $\mathrm{p}<0.001$; CXCL10 $\mathrm{p}<0.001)$. Biomarker levels in both plasma and saliva in subjects with malaria and without malaria were correlated and a significant relationship was found between Ang -2 and CXCL10 which could be used to predict malaria severity $(p=0.001$ for Ang- 2 and $\mathrm{p}<0.01$ for CXCL10). Low Ang-1 and high Ang-2 in both plasma and saliva were significantly associated with increased risk of malaria severity: Ang-1, 2741.04 (1785.85-3582.68), $\mathrm{p}<0.009 ; \quad$ Ang-2, $3508.82 \quad$ (2139.61-5091.633.9), $\mathrm{p}<0.001$ and Ang-1, 720.27 (439.82-1086.74); 16.98 (10.0833.26), ( $p<0.001$ for all). Finally, Ang-2 was informative when combined with CXCL10 to predict malaria severity.

Conclusion These results provide insight into the use of saliva for a non-invasive diagnostic method and demonstrate that Ang-2 combined with CXCL10 is a promising predictive biomarker of malaria severity.

\section{PO 8369 BURULI ULCER: PATTERN OF PRESENTATION IN A NIGERIAN HOSPITAL}

${ }^{1}$ Chukwuanugo Ogbuagu*, ${ }^{2}$ Ifeoma Enweani, ${ }^{3}$ Agu Maurice, ${ }^{1}$ Ekenechukwu Ogbuagu, ${ }^{1}$ Obiageli Emelumadu. ${ }^{1}$ Nnamdi Azikiwe University Teaching Hospital, Nnewi, Nigeria; ${ }^{2}$ Nnamdi Azikiwe University, Awka, Nigeria; ${ }^{3}$ Nnewi Diocesan Hospital, Nnewi, Nigeria

10.1136/bmjgh-2019-EDC.76 